eventually lead to a backlash which will destroy confidence and the necessary need for vigilance and referral of suspected cases of the syndrome. From its nature, this confidence can be built up only slowly, after years of painstaking work, but will be rapidly lost by a few injustices. - I am, etc.,

JAMES K. SARSFIELD

Department of Paediatrics and Child Health,

University of Leeds

\section{Unusual Complication of Use of Sengstaken-Blakemore Tube}

SIR,-We present, below, a previously undescribed hazard of the use of the Sengstaken-Blakemore tube.

The patient was a 55-year-old male alcoholic who presented with bleeding oesophageal varices. During the course of management a SengstakenBlakemore tube was passed and inflated with a
Gastrografin and saline solution. ${ }^{2}$ The position of Gastrografin and saline solution. ${ }^{12}$ The tube was checked radiologically.

Despite sedation the patient was restless and Despite sedation the patient was restless and
succeeded in pulling the tube so that the oesophageal balloon was partly out of his mouth. The oesophageal balloon was immediately defiated, but no Gastrografin could be aspirated from the gastric balloon. The tube was passed back to the stomach and restored to the traction position. An $x$-ray (fig. 1) showed the gastric balloon still inflated and in position, but the distance between it and the now malpositioned oesophageal balloon showed that the patient's sudden traction had pulled the that the patient's sudden traction had pulled the gastric balloon a considerable distance down the tube. Gastrografin injected via the gastric balloon's filling lumen did not increase the balloon's size, nor could it be reaspirated, showing that the bal

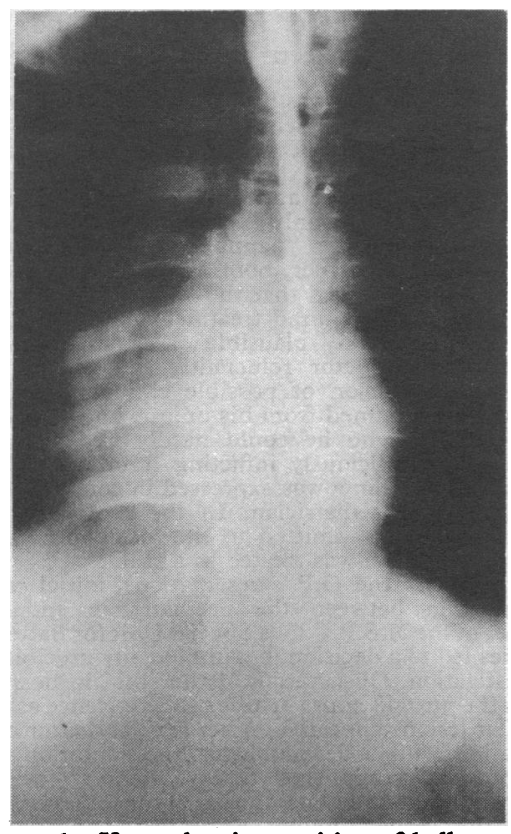

TG. 1-X-ray showing position of balloons after traction by patient.

Later the patient again pulled on the tube and, despite the inflation of the gastric balloon, he succeeded in extracting it, fortunately without damage to the oesophagus. Fig. 2 shows that the gastric to the oesophagus. Fig. 2 shows that the gastric
balloon had now been forced to the tip of the balloon had now been forced to the tip of the is revealed by the two holes of the filling lumen just is revealed by the two holes of the
below the oesophageal balloon.

This case illustrates that the gastric balloon of the Sengstaken-Blakemore tube can be forced away from its filling lumen to a position where it can be neither filled nor deflated. This was done in this case by a restless patient, but there is a danger of the

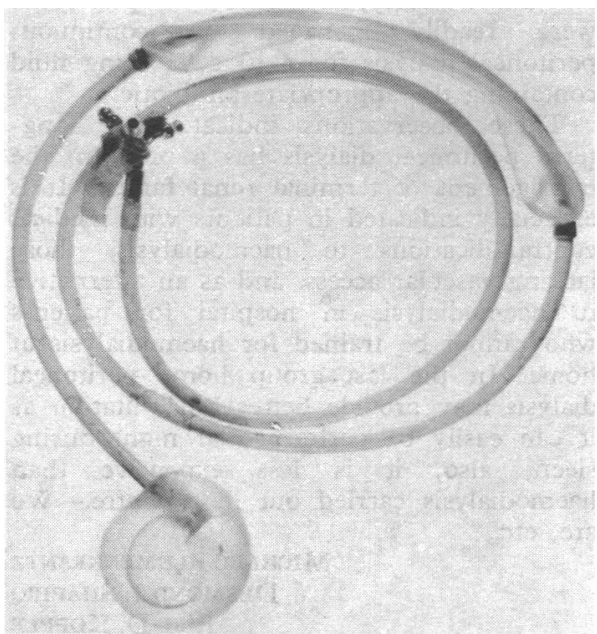

FIG. 2.-Tube after removal by patient.

same complication arising from traction applied therapeutically to the tube-a procedure criticized on other grounds by Pitcher. ${ }^{3}$ This is potentially dangerous for in this situation there may be no way in which the gastric balloon can be safely removed without a laparotomy and gastrotomy.

To some extent the dangers of this complication may be minimized by light sedation, but too heavy sedation increases the risks of inhalation of secretions. The problem could be avoided by redesigning the tube with continuous rubber balloons on the lines of the Foley urinary catheter balloon.-We are, etc.,

C. J. HAWKEY

Northwick Park Hospital

N. R. PEDEN

Harrow, Middlese

1 Read, A. B., Dawson, A. M., Kerr, D. N. S., Turner, M. D., and Sherlock, S., British 2 McPherson, A. I. S., Scottish Medical fournal, 3 Pitcher, J. L., Gastroenterology, 1971, 61, 291.

\section{Apathetic T-3 Toxicosis}

SIR,-Drs. P. D. Fairclough and G. M. Besser (2 March, p. 364) rightly emphasize the occult nature of many cases of hyperthyroidism, be it due to thyroxine or triiodothyronine. Their patient is yet another example of the situation in which a prolonged period of ill health precedes a firm diagnosis of overt hyperthyroidism.

There is evidence that a prethyrotoxic state exists. In elderly subjects, in whom the cardiovascular system is relatively vulnerable, symptoms and signs referable to the heart and circulation may be due to this form of occult hyperthyroidism. The diagnosis can be suspected when only the serum protein-bound iodine is persistently raised and in eight such cases ${ }^{1}$ each developed obvious hyperthyroidism. A similar syndrome may well arise with triiodothyronine, though chemically the diagnosis is even more difficult to establish, as Drs. Fairclough a'nd Besser have shown.-I am, etc.,

\section{London $\mathbf{W} .1$}

CECIL SYMONS 1 Symons, C., Richardson, P. J., and Wood, J. B.,
Patients' Attitudes to Medical Students in General Practice

SIR,-As a student I read with great interest Dr. H. J. Wright's observations (2 March, p. 372) and would like to add some of my own.

I have recently completed an initial course with a group practice in Manchester. Though this was apparently not recognized by its patients as a teaching practice, I very rarely felt that my presence was in any way resented by the patients. On the contrary, many expressed surprise that they might be expected to object (this discoun's the small number of more senior patients who feared that their life-long doctor was being replaced by a young unknown entity and whose fears were rapidly dispelled). Nevertheless, I would have considered the courteous and correct opening to any consultation to be to introduce those present, if necessary explaining the capacity in which they are present, and to ascertain whether the patient objects to their presence. I should have expected such practice to be a common, indeed natural. procedure and was surprised that Dr. Wright thought it necessary to make such a recommendation.

My experience and that of other students, though somewhat limited, indicates that the manner of introduction may have a onsiderable effect on acceptance by the patient. The term "medical student" implies that the patient is being used as a teaching object, an idea which the presence of two or more students may potentiate, and any such feeling might become a barrier to doctorpatient rapport. The patient-doctor relationship is more personal in general practice than in hospital and therefore to allow the presence of a student at a consultation is an even greater privilege granted by the patient. I believe that the presence of more than one student is an abuse of this privilege.

The introduction of the student as a "student doctor" or, with a little poetic licence, a "young doctor," though meaningless terms, considerably aided acceptance by the patient and also helped to encourage participation by the student. While I was under the guise of a "young doctor frmm the Infirmary" my presence was not objected to during consultation or physical examination, nor indeed was my examination of the patient (which included an internal examination where indicated). The only occasions on which I encountered uneasiness involved patients with psychosocial problems wishing to discuss them with their own doctor; I believe the unease was due to intrusion by another individual rather than specific objection to a student. In such a situation I found it necessary to withdraw and become a very passive observer or to leave the consulting room altogether. -I am, etc.

PHIL G. WIIES

Manchester

\section{Acute Pancreatitis and Diabetic} Ketoacidosis in Hypothermia

SIR,-May we support Dr. R. E. G. Sloan's comments (9 Februarv, p. 245) concerning the need to use arterial blood samples for all biochemical estimations made on hypothermic patients. This has always been our 